\title{
Surface functionalized spherical nanoparticles: an optical assessment of local chirality
}

\author{
Jamie M. Leeder, Henryk T. Haniewicz and David L. Andrews \\ School of Chemical Sciences, University of East Anglia, Norwich NR4 7TJ, United Kingdom
}

\begin{abstract}
Electromagnetic radiation propagating through any molecular system typically experiences a characteristic change in its polarization state as a result of light-matter interaction. Circularly polarized light is commonly absorbed or scattered to an extent that is sensitive to the incident circularity, when it traverses a medium whose constituents are chiral. This research assesses specific modifications to the properties of circularly polarized light that arise on passage through a system of surface-functionalized spherical nanoparticles, through the influence of chiral molecules on their surfaces. Nonfunctionalized nanospheres of atomic constitution are usually inherently achiral, but can exhibit local chirality associated with such surface-bound chromophores. The principal result of this investigation is the quantification of functionally conferred nanoparticle chirality, manifest through optical measurements such as circularly polarized emission. The relative position of chiral chromophores fixed to a nanoparticle sphere are first determined by means of spherical coverage coordinate analysis. The total electromagnetic field received by a spatially fixed, remote detector is then determined. It is shown that bound chromophores will accommodate both electric and magnetic dipole transition moments, whose scalar product represents the physical and mathematical origin of chiral properties identified in the detected signal. The analysis concludes with discussion of the magnitude of circular differential optical effects, and their potential significance for the characterization of surface-functionalized nanoparticles.
\end{abstract}

Keywords: Quantum Electrodynamics, Chirality, Functionalized Nanoparticles, Photon Emission, Stokes Vector

\section{INTRODUCTION}

Chirality is a property attributed to two otherwise identical entities that are non-superimposable upon their mirror image. It is a characteristic of physical materials and optical radiation featuring in a diverse range of prominent research fields including drug development, chemical catalysis and nanoscale metamaterials. In many of the material applications, it is vitally important to distinguish, quantify and ultimately separate chiral matter into pure samples that exhibit identical chemical properties. Fortunately, a number of powerful techniques have been developed that enable such analysis; for example in circular dichroism spectroscopy a chiral molecule's capacity to exhibit a differential response based on the handedness of the input light is manifest in a quantifiable difference in the absorption rate of left- and right-handed photons. ${ }^{1-3}$ In the time-reverse process, it follows that chiral discrimination is also achievable through measurements of spontaneous emission by circular polarized luminescence spectroscopy. ${ }^{3-6}$ In fact, regarding the wealth of information that can be discerned from polarization resolved optical measurements, there exists a renewed interest in the inherent links between molecular chirality and the helicity of light emitted from optically active molecular chromophores. ${ }^{7,8}$

To address, in detail, specific relationships between various practical measures of chirality, the simplest optical process through which material chirality is conferred into an optical field is spontaneous circularly polarized photon emission by a single chiral molecule. This investigation represents a logical extension in which we consider a more complex, yet highly prevalent form of chromophore in the form of surface coated nanoparticles (SCN). Essentially an SCN consists of a shaped nanoparticle core covered in a thin layer of organic or inorganic material, the most prominent examples being silica-coated gold or iron oxide. These nanoparticle materials are commonly developed for their diverse biomedical applications, yet they are equally recognised as important technology in analytical science where SCNs are employed as molecular sensors and biomarkers. ${ }^{9-13}$ For the present investigation, surface coated spherical nanoparticles are considered due to their uniformity in size and shape in all directions from a local origin, thus their lack of chiral properties - essentially, nanoparticle spheres as a result of their inherent symmetry are characterized as macroscopically, or globally achiral. It is proposed that incorporation of chiral molecular chromophores on a nanosphere surface will confer local chiral effects on such a SCN. Further, such effects should prove detectable and quantifiable through polarization resolved measurements of the emitted optical field. 
In the theoretical investigation to follow, the details of an emitter-detector system are first detailed, establishing the nature of an arbitrary surface coated nanosphere functionalized with chiral, molecular chromophores. The relative positioning of chromophores on the sphere surface will be established by use of optimal spherical covering coordinates. Radiative emission from the sphere, sourced from the chiral chromophores, will be subject to polarization-resolved measurements and the results will be utilized to characterize conferred chiral properties. A theoretical formulation of all associated electromagnetic fields will be achieved within the fully quantized formalism of quantum electrodynamics (QED). The effect on the recorded field as influenced by key variables including the number of surface bonded chromophores per sphere and the separation distance between the emitter and the detector will be determined. The latter is of particular interest, allowing both long-range and local field effects to be considered in detail.

\section{THEORETICAL METHODS}

In order to assess chiral emission from a surface functionalized nanosphere it is important to first establish both the physical and optical properties of the emitter. A single nanosphere of radius $r$ is coated with a total number of chromophores $n$, such that at any point in time any of them might be found in an excited electronic state. All chromophores are evenly distributed around the nanosphere surface with the exact position of each being based upon coordinates of the optimal covering of a sphere in 3-dimensions as reported by Sloane et al-see figure $1 .{ }^{14}$ Such coordinates formally determine the position vectors of each chromophore relative to the sphere centre, and they also define the orientation of electronic and magnetic transition dipole moments. For each chromophore, the latter transition moments are assumed to be parallel to each other, pointing outwards from the center of the sphere. It is further supposed that individual chromophores undergo no optical interactions with each other, and that the nanoparticle as a whole is entirely transparent to any optical emission sourced from the attached emitters.

\begin{tabular}{|c|c|c|c|}
\hline $\begin{array}{c}\text { Chromophore } \\
\text { number }\end{array}$ & X-coordinate & Y-coordinate & Z-coordinate \\
\hline 1 & 0.8507 & 0.0000 & -0.5257 \\
\hline 2 & 0.5257 & -0.8507 & 0.0000 \\
\hline 3 & 0.0000 & -0.5257 & 0.8507 \\
\hline 4 & 0.8507 & 0.0000 & 0.5257 \\
\hline 5 & -0.5257 & -0.8507 & 0.0000 \\
\hline 6 & 0.0000 & 0.5257 & -0.8507 \\
\hline 7 & -0.8507 & 0.0000 & -0.5257 \\
\hline 8 & -0.5257 & 0.8507 & 0.0000 \\
\hline 9 & 0.0000 & 0.5257 & 0.8507 \\
\hline 10 & -0.8507 & 0.0000 & 0.5257 \\
\hline 11 & 0.5257 & 0.8507 & 0.0000 \\
\hline 12 & 0.0000 & -0.5257 & -0.8507 \\
\hline & & & \\
\hline
\end{tabular}

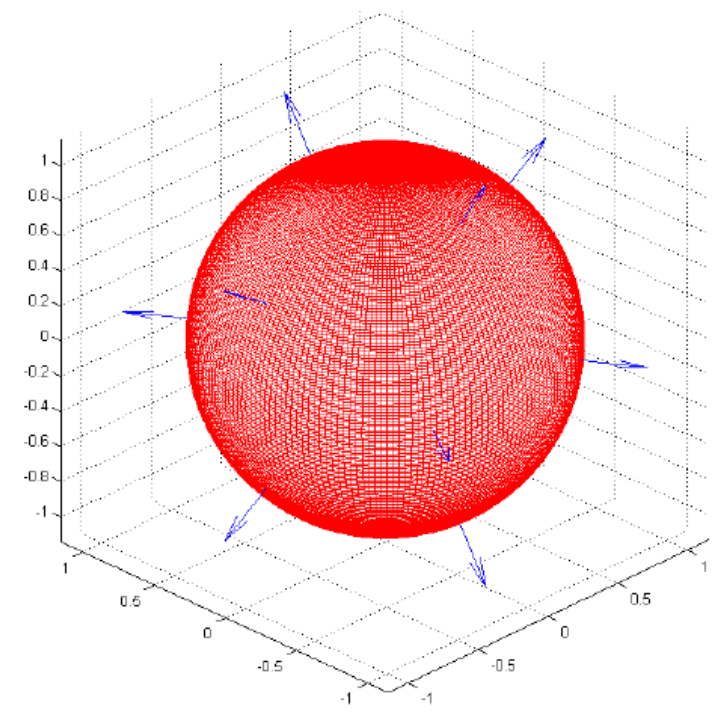

Figure 1 - Optimal covering coordinates and diagrammatic representation of a functionalized nanopshere with 12 evenly distributed chromophores. The chromophores form a regular icosahedron on the surface of a sphere with dimensions presented in units of nanoparticle radius $r$.

Regarding the optical field measurement, the polarization of a single quantized mode from any emitter is routinely quantified in terms of four Stokes parameters $S_{n}$, each of which directly relates to wave intensities measured in different orthogonal bases. ${ }^{15}$ In considering the differential emission of both left- and right-handed optical emission from a chiral emitter the most significant operator is $S_{3}$, commonly presented in the following form;

$$
S_{3} \equiv I_{R}-I_{L}=2 \operatorname{Im}\left[\bar{E}_{x} E_{y}\right] \equiv \operatorname{Im}\left[\bar{E}_{x} E_{y}-\bar{E}_{y} E_{x}\right] \text {. }
$$


In equation (1) $I_{R}$ and $I_{L}$ represent the field intensities of right- and left-handed, circularly polarized emission respectively, and each $\mathbf{E}$ is a complex vector associated with the electric field. For an electronically excited transmitter whose chirality permits a radiative decay transition to be simultaneously allowed by both E1 and M1 selection rules, the total electric field is expressible as:

$$
E_{j}=-\mu_{i} V_{i j}-c^{-1} m_{i} U_{i j}
$$

where $\boldsymbol{\mu}$ is the electric dipole moment of the source transmission and $\mathbf{m}$ represents a magnetic dipole equivalent. These two terms suffice for the identification of conferred chirality. Within a fully quantized theoretical formalism, derivation of the second-rank E1-E1 and E1-M1 coupling tensors $V_{i j}$ and $U_{i j}$ respectively, is achieved by summing the quantum amplitudes of emitted, circularly polarized photons of arbitrary propagation and polarization vector; ${ }^{16}$

$$
\begin{aligned}
& V_{i j}=\frac{\exp (i k R)}{4 \pi \varepsilon_{0} R^{3}}\left[(1-i k R)\left(\delta_{i j}-3 \hat{R}_{i} \hat{R}_{j}\right)-k^{2} R^{2}\left(\delta_{i j}-\hat{R}_{i} \hat{R}_{j}\right)\right] \\
& U_{i j}=\frac{\exp (i k R)}{4 \pi \varepsilon_{0}} \varepsilon_{i j k} \frac{\hat{R}_{k}}{R^{3}}\left(-i k R-k^{2} R^{2}\right)
\end{aligned}
$$

Here, $k$ represents the magnitude of the emission wavevector and $R$ is the distance between a chromophore emitter and the detector. Upon substitution of the tensors in equation (3) into equation (2), it is possible to determine the $x$ and $y$ components of the emerging field as required by equation (1), where the former for example is expressible as:

$$
E_{x}=-\left(\mu_{x} V_{x x}+\mu_{y} V_{y x}+\mu_{z} V_{z x}+c^{-1} m_{y} U_{y x}+c^{-1} m_{z} U_{z x}\right)
$$

It is worth noting that equation (4) will account for an arbitrary point detector, positioned at a distance $R^{\prime}$ from the center of the nanoparticle, see Fig 2. The detector will measure propagating radiation along the z-axis of the emission, thus $\hat{R}_{k}=\hat{R}_{z}$, hence $U_{z x}=0$ in equation (4). Otherwise the magnitude of all unit displacement vectors that feature in both the E1-E1 and E1-M1 tensors depend on the position of each chromophore on the nanoparticle emitter relative to the detector as determined by use of the spherical covering coordinates.

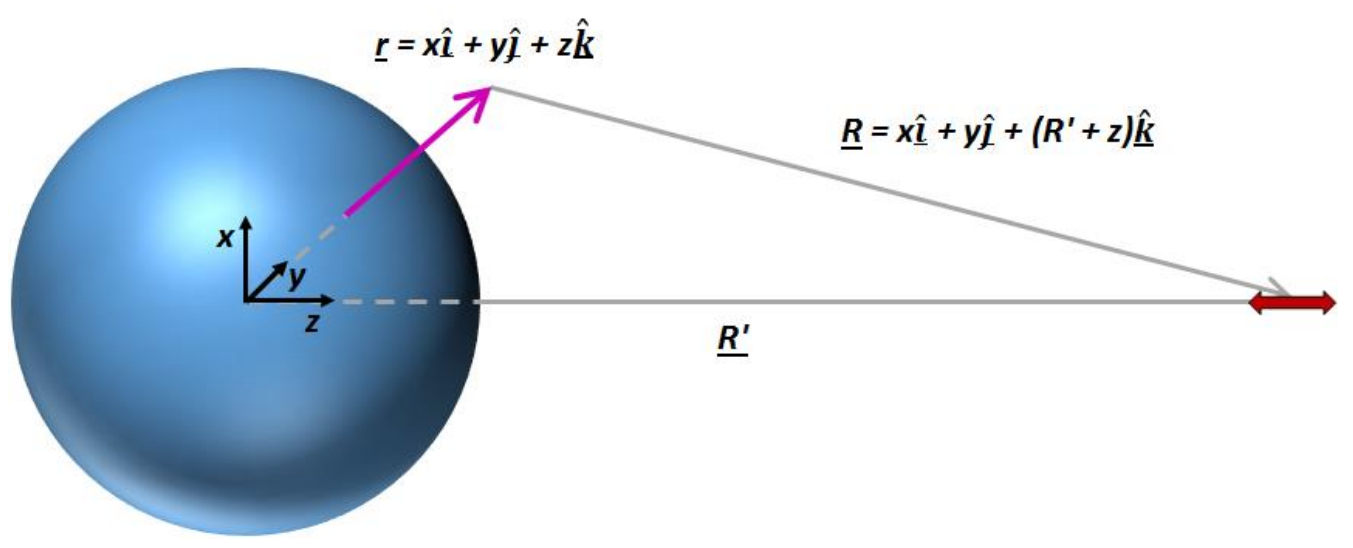

Figure 2 - Illustration of the proposed nanoparticle emitter and point detector system. In this example a single chromophore (surface normal arrow) is attached to the surface of the nanosphere. The chromophore displacement relative to a point detector (double arrow) is determined based on optimum spherical covering coordinates.

\section{RESULTS}

The $S_{3}$ parameter of an emerging electric field from a single molecular chromophore follows derivation of expressions for $E_{x}$ and $E_{y}$ and subsequent substitution into equation (1). The overall result is expressible as the sum of both near-zone (NZ) and far-zone (FZ) terms that describe how the system responses in two distinct distance regimes. The NZ result 
becomes most significant in any case where the distance between the emitter and the detector is substantially smaller than the wavelength of emitted radiation, i.e. $R \ll \lambda$. It transpires in the near-zone that $S_{3}$ varies with a dependence on $R^{-3}$, specifically:

$$
S_{3}^{N Z}=\frac{k^{3}}{8 \pi^{2} \varepsilon_{0}^{2} R^{3}}\left[\mu_{y}\left(\mu_{x} \hat{R}_{x}^{2}+\mu_{y} \hat{R}_{x} \hat{R}_{y}+\mu_{z} \hat{R}_{x} \hat{R}_{z}\right)-\mu_{x}\left(\mu_{y} \hat{R}_{y}^{2}+\mu_{x} \hat{R}_{x} \hat{R}_{y}+\mu_{z} \hat{R}_{y} \hat{R}_{z}\right)\right]
$$

Of interest, it is worth noting that all terms in equation (5) feature a product of two electric transition dipole moments. In comparison, all terms that determine the system response in the far-zone regime where $R \gg \lambda$ exhibit a dependence on $R$ 2 and feature a product of E1 and M1 transition moments:

$$
\begin{aligned}
S_{3}^{F Z} & =\frac{k^{4}}{\left(4 \pi \varepsilon_{0} R\right)^{2} c}\left[\mu_{x}\left(\tilde{m}_{x} \hat{R}_{z}-\tilde{m}_{x} \hat{R}_{x}^{2} \hat{R}_{z}-\tilde{m}_{y} \hat{R}_{x} \hat{R}_{y} \hat{R}_{z}\right)+\mu_{y}\left(\tilde{m}_{y} \hat{R}_{z}-\tilde{m}_{y} \hat{R}_{y}{ }^{2} \hat{R}_{z}-\tilde{m}_{x} \hat{R}_{x} \hat{R}_{y} \hat{R}_{z}\right) .\right. \\
& \left.-\mu_{z}\left(\tilde{m}_{x} \hat{R}_{x} \hat{R}_{z}^{2}+\tilde{m}_{y} \hat{R}_{y} \hat{R}_{z}^{2}\right)\right]
\end{aligned}
$$

The presented results are general. Consequently, variation in the $S_{3}$ signal based on any number of independent variables - including the number of chromophores attached to the nanosphere emitter, or the distance between the nanosphere emitter and the point detector - requires assignment of numerical values to experimental parameters that feature in equations (5) and (6). For present purposes, all E1 transition moments are assumed to have a magnitude of 1 D - each M1 transition moment is smaller by a factor of the fine-structure constant. The nanosphere radius, $r$ is arbitrarily set at $1 \mathrm{~nm}$ and the number of chromophores attached to the spherical surface is investigated within the range $n=4$ and $n=20-$ the total field is the sum of contributions from each molecular emitter. The overall dependence on the detected signal to emitter-detector separation is shown in Fig 3.

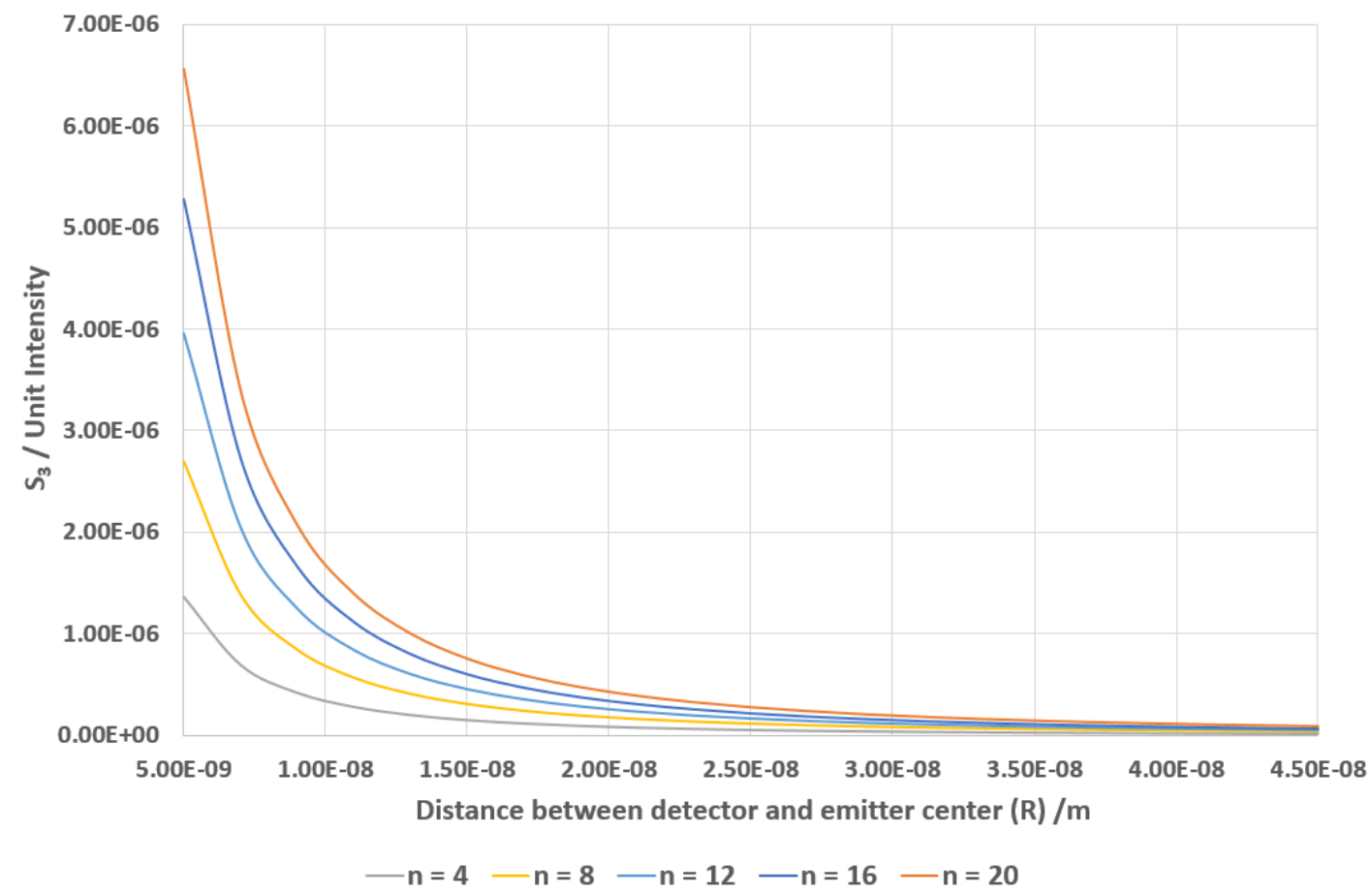

Figure 3 - The $S_{3}$ signal from a surface functionalized nanosphere. Each series represents an emitter functionalized with different numbers of evenly distributed molecular chromophores. In these example results, the wavelength of the emitted radiation is arbitrarily set at $700 \mathrm{~nm}$. 
The calculated $S_{3}$ signal is clearly most significant at very short emitter-detector separations, particularly in systems that feature a large number of surface attached chromophores. Physically, it can be understood that at a large separation, detection of radiation from molecular emitters fail to register the chiral nature of the source. In contrast, at shorter distances, local chiral effects can indeed be observed. Shown in Fig. 3, all nanoemitter systems exhibit a reduction in the recorded $S_{3}$ radiant intensity of at least $70 \%$ beyond an emitter-detector separation of $10 \mathrm{~nm}$. Preliminary investigations suggest that such loses in the recorded signal are typical for any system where the detector is placed away from the source at a distances that exceed ten times the emitter radius. An interesting, and not obviously apparent point of discussion concerns how the reported dependence on small emitter-detector separation is not attributable to the magnitude of the previously presented near-zone result at low values of $R$. As the total $S_{3}$ signal is calculated as the sum of equations (5) and (6), it would be expected that the NZ contribution dominates when the detector and emitter are close to each other and that the sum of all FZ terms becomes more prominent at larger values of $R$. In fact, at all times, the NZ result is smaller than the FZ equivalent by multiple orders of magnitude. This disparity indicates that the total calculated $S_{3}$ parameter, determined at any emitter-detector separation, can to a good approximation be expressed simply as equation (6).

\section{DISCUSSION}

The results of this investigation show how it is possible to confer a degree of local chirality upon an inherently achiral nanoparticle sphere by functionalizing the sphere's surface with evenly distributed, optically active molecular chromophores. Theoretical calculations based upon the experimental technique of Stokes polarimetry have been utilized to verify molecular chirality by use of an optical method of measurement. Specifically, quantification of the chiral nature of an emerging optical field is determined by use of the $S_{3}$ parameter which considers the differential emission of both leftand right-handed circularly polarized light. For the systems in this current study, the $S_{3}$ signal is recorded by an arbitrary point detector and found to be significant only in the immediate vicinity surrounding the emitter, i.e. at emitter-detector separations similar in magnitude to the nanosphere radius. Increased signals are noted when greater numbers of attached chromophores per nanosphere are considered, and for systems that incorporate chromophores with large electric and magnetic transition dipole moments - the $S_{3}$ magnitude increases approximately as a square of any increase in the E1 and M1 moments. A number of further investigations are expected to follow that further explore the assumptions considered in this initial research. To begin, in order to more faithfully represent practical experimental systems, it is necessary to consider in these calculations the various mechanisms by which the nanoparticle spheres and their attached chromophores might mutually interact. It is likely, for example, that multiple chromophores attached to a single nanoparticle will be in close enough proximity to exhibit optical scattering effects and/or non-radiative radiation-matter interactions such as Förster resonance energy transfer. For now, optimum covering coordinates have been employed to model chromophores evenly distributed around the nanosphere surface; however, less symmetrical arrangements of such chromophores will also likely result in modified emission properties. In such cases, the orientation of the nanospheres relative to the detector will need to be addressed - by extension, rotation of the spheres will also need to be considered. Beyond localized effects concerning the properties of individual spheres, there exists further scope to explore how multiple surface functionalized emitters interact with each other. Indeed, experimental devices based upon this research could further consider arrays of surface coated nanoparticles immobilized on metal surfaces where additional plasmonic effects may contribute to enhanced field emission.

\section{ACKNOWLEDGEMENTS}

The authors are grateful to the Engineering and Physical Sciences Research Council (EPSRC) for financing this work.

\section{REFERENCES}

[1] Ranjbar, B., Gill, P., "Circular Dichroism Techniques: Biomolecular and Nanostructural Analyses- A Review," Chem. Biol. Drug Des. 74(2), 101-120 (2009).

[2] Polavarapu, P. L., "Why is it important to simultaneously use more than one chiroptical spectroscopic method for determining the structures of chiral molecules?," Chirality 20(5), 664-672 (2008).

[3] Brittain, H. G., "Applications of chiroptical spectroscopy for the characterization of pharmaceutical compounds," J. Pharm. Biomed. Anal. 17(6), 933-940 (1998). 
[4] Carr, R., Evans, N. H., Parker, D., "Lanthanide complexes as chiral probes exploiting circularly polarized luminescence," Chem. Soc. Rev. 41(23), 7673 (2012).

[5] Riehl, J. P., Richardson, F. S., "Circularly polarized luminescence,” Methods Enzymol. 226, 539-552 (1993).

[6] Riehl, J. P., Richardson, F. S., "Circularly polarized luminescence spectroscopy,” Chem. Rev. 86(1), 1-16 (1986).

[7] Tang, Y., Cohen, A. E., “Optical Chirality and Its Interaction with Matter,” Phys. Rev. Lett. 104(16), 163901 (2010).

[8] Bliokh, K. Y., Nori, F., “Characterizing optical chirality,” Phys. Rev. A 83, 021803 (2011).

[9] Nguyen, D. T., Kim, D.-J., Kim, K.-S., "Controlled synthesis and biomolecular probe application of gold nanoparticles,” Micron 42(3), 207-227 (2011).

[10] Ruedas-Rama, M. J., Walters, J. D., Orte, A., Hall, E. A. H., "Fluorescent nanoparticles for intracellular sensing: A review," Anal. Chim. Acta 751, 1-23 (2012).

[11] Valentini, P., Pompa, P. P., "Gold nanoparticles for naked-eye DNA detection: smart designs for sensitive assays,” RSC Adv. 3(42), 19181 (2013).

[12] Jiang, S., Win, K. Y., Liu, S., Teng, C. P., Zheng, Y., Han, M.-Y., "Surface-functionalized nanoparticles for biosensing and imaging-guided therapeutics," Nanoscale 5(8), 3127 (2013).

[13] Wu, W., Wu, Z., Yu, T., Jiang, C., Kim, W.-S., "Recent progress on magnetic iron oxide nanoparticles: synthesis, surface functional strategies and biomedical applications," Sci. Technol. Adv. Mater. 16(2), 023501 (2015).

[14] Hardin, R. H., Sloane, N. J. A., Smith, W. D., "Spherical Codes,” Online Preparation Available: http://www.neilsloane.com/coverings/dim3 (1994).

[15] Goldstein, D. H., [Polarized Light, 3rd ed.], CRC Press, Boca Raton (2011).

[16] Daniels, G. J., Jenkins, R. D., Bradshaw, D. S., Andrews, D. L., "Resonance energy transfer: The unified theory revisited," J. Chem. Phys. 119, 2264-2274 (2003). 